\title{
Polymer Inclusion Membrane Containing a Tripodal Diglycolamide (T-DGA) Ligand: Actinide Ion Uptake and Transport Studies
}

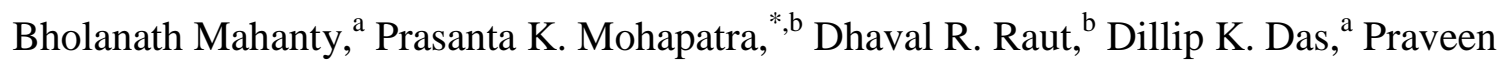
G. Behere, ${ }^{\mathrm{a}}$ Mohammed Afzal, ${ }^{\mathrm{a}}$ and Willem Verboom ${ }^{\mathrm{c}}$

${ }^{a}$ Advanced Fuel Fabrication Facility, B. A. R. C., Tarapur - 401502, India

${ }^{b}$ Radiochemistry Division, B. A. R. C., Mumbai - 400085, India

${ }^{c}$ MESA+ Institute for Nanotechnology, University of Twente, Enschede, The Netherlands

*Corresponding author. Email: mpatra@barc.gov.in

\section{ELECTRONIC SUPPORTING INFORMATION}




\section{EXPERIMENTAL}

1.1 Radiochemical purity of the radiotracers

All radionuclides were used after checking their radiochemical purities.

${ }^{233} \mathrm{U}:$

${ }^{233} \mathrm{U}$ was purified using an anion exchange method (Dowex $1 \mathrm{x} 8, \mathrm{Cl}^{-}$form resin was used) as reported previously to get rid of its major decay products such as ${ }^{228} \mathrm{Th}$ (daughter product of ${ }^{232} \mathrm{U}$ which is present in small quantity along with ${ }^{233} \mathrm{U}$ ) and its daughter products. It is well known that in $6 \mathrm{M} \mathrm{HCl}$, anionic complexes of $\mathrm{U}$ are held onto the column while ${ }^{232} \mathrm{Th}$ and its daughter products pass through (S.S. Rattan, et al., J. Radioanal. Chem., 67(1981)95). The loaded U was subsequently eluted using dilute HCl. Alpha spectrometry of the purified ${ }^{233} \mathrm{U}$ stock suggested to be free from ${ }^{228} \mathrm{Th}$ and its daughter products.

$\underline{\mathrm{Pu}}$ :

$\mathrm{Pu}$ (mixture of isotopes but mainly ${ }^{239} \mathrm{Pu}$ ) was purified from its major decay product ${ }^{235} \mathrm{U}$ and ${ }^{241} \mathrm{Am}$ (from beta decay of ${ }^{241} \mathrm{Pu}$ ) by TTA extraction (using 0.5 M TTA in xylene) from an aqueous phase containing $1 \mathrm{M} \mathrm{HNO}_{3}$. Under these conditions, $\mathrm{Am}^{3+}$ and $\mathrm{UO}_{2}{ }^{2+}$ are not extracted while $\mathrm{Pu}^{4+}$ is extracted quantitatively. $\mathrm{Pu}$ was subsequently stripped by $8 \mathrm{M} \mathrm{HNO}_{3}$ and the alpha spectrometry of the purified stock was carried out to rule out the presence of the impurities.

${ }^{241} \mathrm{Am}:$

${ }^{241} \mathrm{Am}$ was purified from its daughter product, ${ }^{237} \mathrm{~Np}$, by first reducing $\mathrm{Np}$ to its +4 state using hydroxylamine hydrochloride at $1 \mathrm{M} \mathrm{HNO}_{3}$ and subsequently extracting $\mathrm{Np}^{4+}$ by TTA (similar to the extraction of $\mathrm{Pu}^{4+}$ mentioned above). The aqueous phase was evaporated to dryness and a few drops of mixture of concentrated $\mathrm{HNO}_{3}$ and $\mathrm{HClO}_{4}(5: 1$ ratio) was added to destroy the organic impurities. Alpha spectrometry of the purified ${ }^{241}$ Am stock was carried out to rule out the presence of the impurities.

${ }^{234} \mathrm{Th}$ :

${ }^{234} \mathrm{Th}$ was obtained from natural uranium which contained a small fraction of ${ }^{234} \mathrm{Th}$ in secular equilibrium with the long-lived parent ${ }^{238} \mathrm{U}$. As Th(IV) does not form any anionic chloro complexes in $\mathrm{HCl}$ medium while $\mathrm{U}(\mathrm{VI})$ forms anionic complexes such as $\mathrm{UO}_{2} \mathrm{Cl}_{3}{ }^{-}$ 
and $\mathrm{UO}_{2} \mathrm{Cl}_{4}{ }^{2-}$, this was used to selectively extract ${ }^{238} \mathrm{U}$ from the mixture of the radionuclides in $6 \mathrm{M} \mathrm{HCl}$ using 30\% Aliquat 336 in chloroform. For quantitative separation, the extraction was repeated several times till the organic phase appeared colorless. The aqueous phase, containing ${ }^{234}$ Th was washed two times with chloroform to remove traces of the organic extractant and diluent. Finally, the aqueous phase was dried under infrared lamp. A few drops of concentrated $\mathrm{HNO}_{3}$ was added and further dried. This step was repeated to convert $\mathrm{Th}^{4+}$ in chloride form to the nitrate form.

\subsection{Oxidation state adjustment}

The oxidation state of $\mathrm{Pu}$ was adjusted to the +4 state by first drying a lone amount of $\mathrm{Pu}$ (about 20 microgram) under an infrared lamp. The dries activity was dissolved in one $\mathrm{mL}$ of $1 \mathrm{M} \mathrm{HNO}_{3}$ and a few drops of $0.005 \mathrm{M} \mathrm{NaNO}_{2}$ solution was added to change the oxidation state from +3 and +6 state to the +4 state. The aqueous solution was contacted with one $\mathrm{mL}$ of $0.5 \mathrm{M}$ TTA in xylene for 30 minutes. The extracted $\mathrm{Pu}^{4+}$ (the unconverted $\mathrm{Pu}^{3+}$ and $\mathrm{PuO}_{2}{ }^{2+}$ are not extracted under these conditions) was subsequently stripped using $7 \mathrm{M} \mathrm{HNO}_{3}$ and kept for subsequent studies involving $\mathrm{Pu}^{4+}$. The oxidation state of $\mathrm{Pu}^{4+}$ was intermittently checked by TTA extraction (log D vs log TTA concentration plot gave slope close to 4$). \mathrm{Pu}^{4+}$ was found to be stable for about one month.

\subsection{Counting of the radiotracer}

For gamma counting:

The counting of gamma ray emitting radiotracers such as ${ }^{241} \mathrm{Am}$ and ${ }^{234} \mathrm{Th}$ was done using a well type $\mathrm{NaI}(\mathrm{Tl})$ scintillator (Para Electronics) counter coupled with a multichannel analyzer (ECIL, India). The peaks under the characteristic gamma ray energies $\left({ }^{241} \mathrm{Am}: 59 \mathrm{kev} ;{ }^{234} \mathrm{Th}: 63 \mathrm{kev}\right.$ and $\left.93 \mathrm{kev}\right)$ were integrated and the background counts under the same energy were subtracted to yield the net counts. Samples were taken in gamma ray counting tubes (dia: $1 \mathrm{~cm}$; height: $15 \mathrm{~cm}$ ) and the sample solution was limited to less than $500 \mu \mathrm{L}$ to restrict errors due to geometry factor to $<1 \%$. The counting statistics error was limited to $<1 \%$ by carrying out long time counting. 
For alpha counting:

The alpha particle emitting radionuclides (such as ${ }^{233} \mathrm{U}$ and ${ }^{239} \mathrm{Pu}$ ) were counted using a liquid scintillation counter (Hidex, Finland). The sample preparation involves adding the required aliquot (about 100-200 $\mu \mathrm{L}$ ) into $5 \mathrm{~mL}$ of scintillator cocktail followed by ultrasonification for 5 minutes to ensure complete extraction of the radionuclide into the toluene phase. The count rate was obtained after background correction and counting for sufficiently long time to ensure statistical errors were $<1 \%$. As toluene based scintillator cocktail was used, the error due to quenching was negligible.

\section{RESULTS}

2.1 Uptake studies with varying T-DGA and NPOE content

Table S1 \%Am Uptake by PIMs Containing Varying Amounts of T-DGA. Feed: 1 $\mathrm{M} \mathrm{HNO}_{3}$

\begin{tabular}{|l|l|l|l|l|}
\hline \multirow{2}{*}{ Time $(\mathrm{h})$} & \multicolumn{4}{|c|}{ \% Am Uptake } \\
\cline { 2 - 5 } & $6.67 \%$ T-DGA & $12.8 \%$ T-DGA & $22.4 \%$ T-DGA & $26.3 \%$ T-DGA \\
\hline 0.5 & 22.6 & 14.3 & 35.0 & 16.3 \\
\hline 1 & 24.2 & 30.1 & 57.9 & 39.3 \\
\hline 2 & 26.4 & 40.5 & 66.9 & 63.1 \\
\hline 3 & 26.5 & 41.4 & 71.9 & 75.7 \\
\hline 4 & 26.6 & 42.6 & 76.5 & 79.5 \\
\hline 24 & 26.6 & 55.4 & 87.6 & 88.4 \\
\hline
\end{tabular}

Table S2 \% Am Uptake by PIMs Containing Varying Amounts of NPOE. Feed: 1 M $\mathrm{HNO}_{3}$

\begin{tabular}{|l|l|l|l|}
\hline \multirow{2}{*}{ Time $(\mathrm{h})$} & \% Am Uptake & \multicolumn{2}{l|}{} \\
\cline { 2 - 4 } & $24.5 \%$ NPOE & $39.6 \%$ NPOE & $56.8 \%$ NPOE \\
\hline 0.5 & 43.4 & 48.3 & 35.0 \\
\hline 1 & 63.3 & 66.7 & 57.9 \\
\hline 2 & 77.7 & 87.1 & 66.9 \\
\hline 3 & 84.7 & 86.6 & 71.9 \\
\hline 4 & 85.4 & 87.8 & 76.5 \\
\hline 6 & 88.2 & 86.9 & 81.6 \\
\hline 24 & 88.7 & 88.8 & 87.6 \\
\hline
\end{tabular}




\subsection{Transport studies}

Figure S1. Transport of $\mathrm{Am}^{3+}$ using T-DGA containing PIMs with varying T-DGA content. Feed: $1 \mathrm{M} \mathrm{HNO}_{3}$; Receiver: $1 \mathrm{M}$ AHIBA.

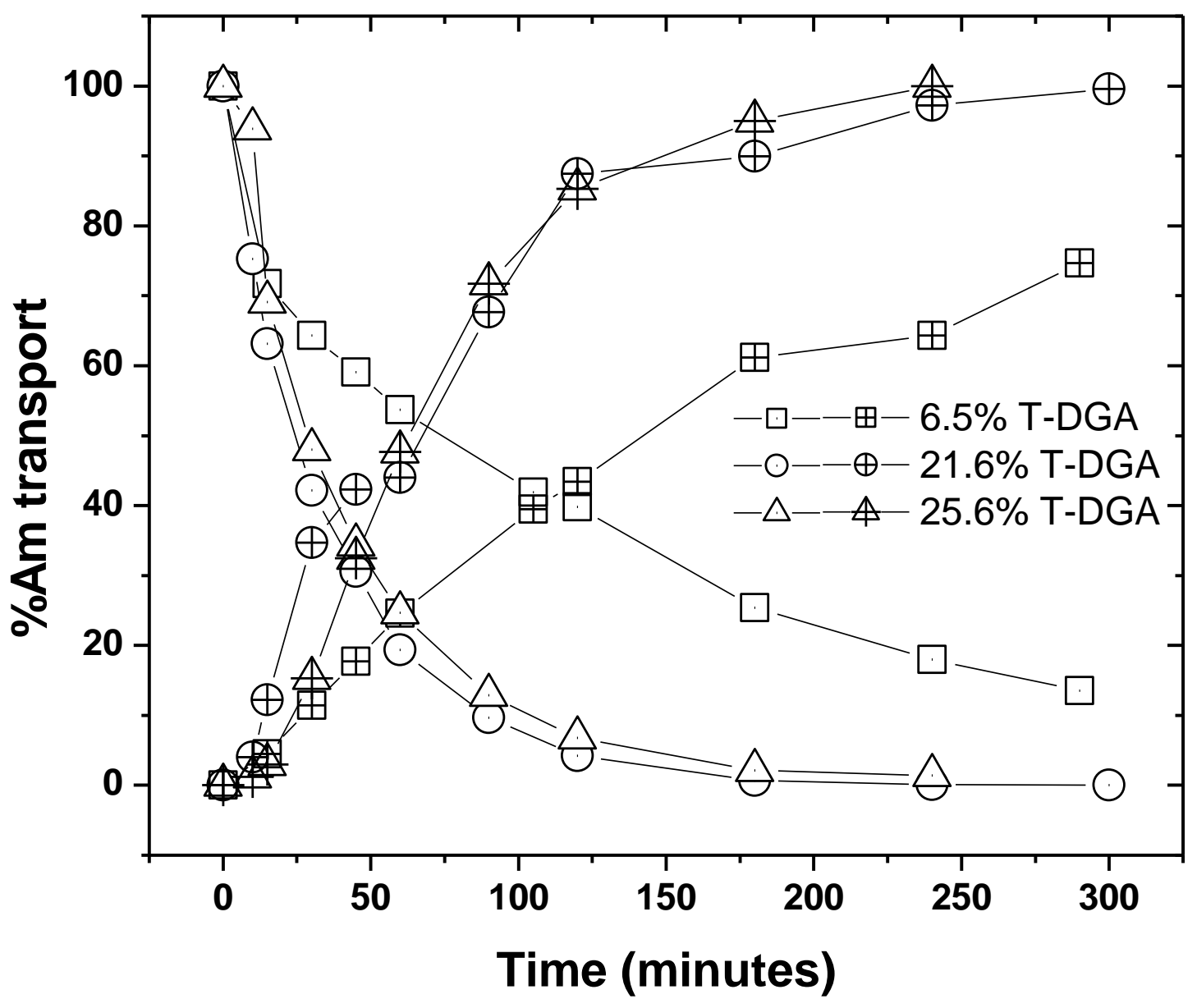


2.3 Determination of diffusion coefficient by lag-time method

In the lag-time method, the time needed for the detection of the metal ion in the receiver compartment was used for the determination of diffusion coefficient as per the following equation (J. Crank in "The Mathematics of Diffusion", 2nd ed., Oxford University Press Inc., New York, 1975, p. 414):

$$
\mathrm{D}_{\text {eff }}=\mathrm{d}_{\mathrm{o}}{ }^{2} \varepsilon / 6 \mathrm{t}_{\mathrm{lag}}
$$

Figure S2. Time-lag plot for Am (III). PIM Composition: 100 mg T-DGA $+200 \mu \mathrm{L}$ $\mathrm{NPOE}+80$ mg CTA.

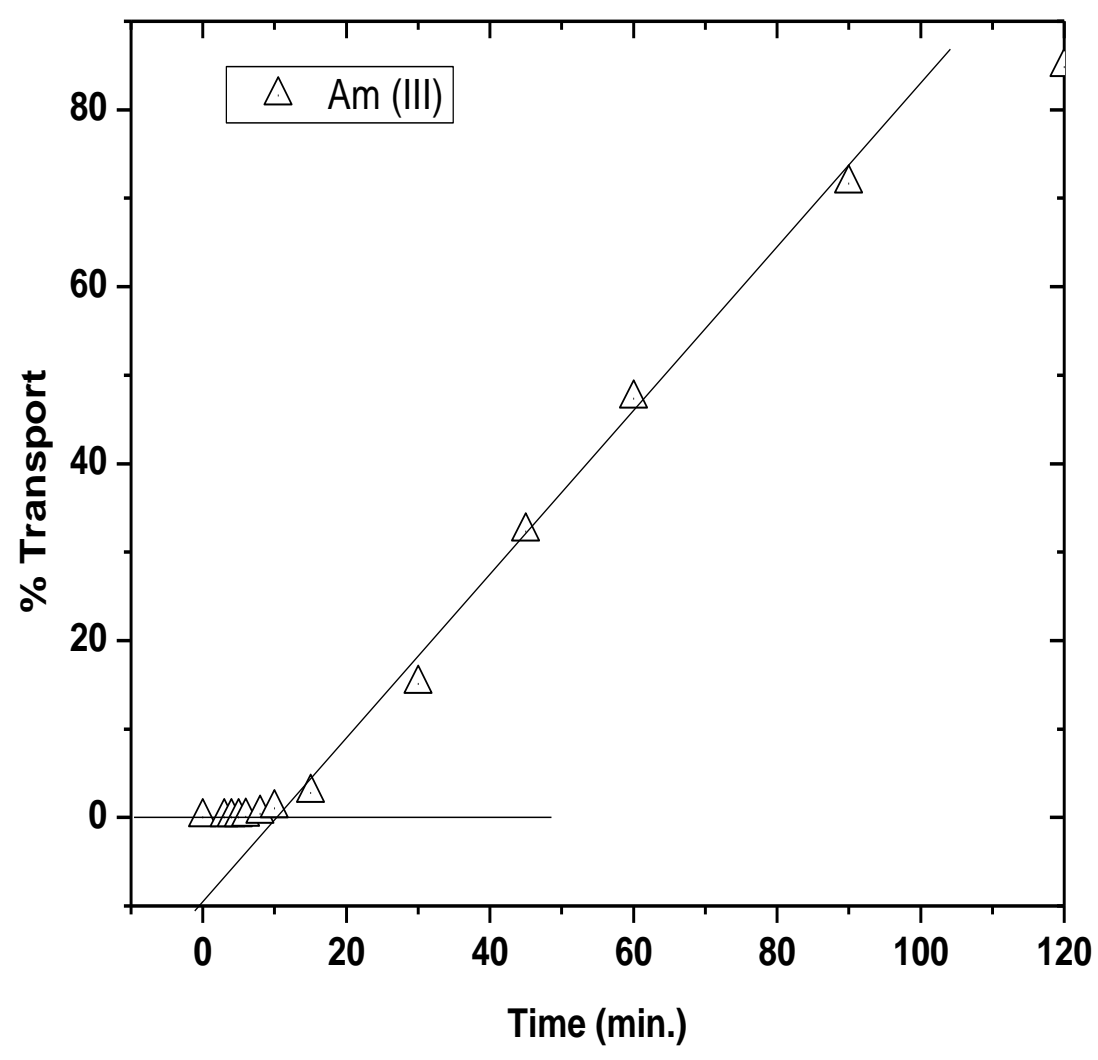




\subsection{Stability of the PIM}

Figure S3. Stability of the T-DGA-containing PIM based on successive $\mathrm{Am}^{3+}$ transport studies. Feed: $1 \mathrm{M} \mathrm{HNO}_{3}$; Receiver: $1 \mathrm{M}$ AHIBA.

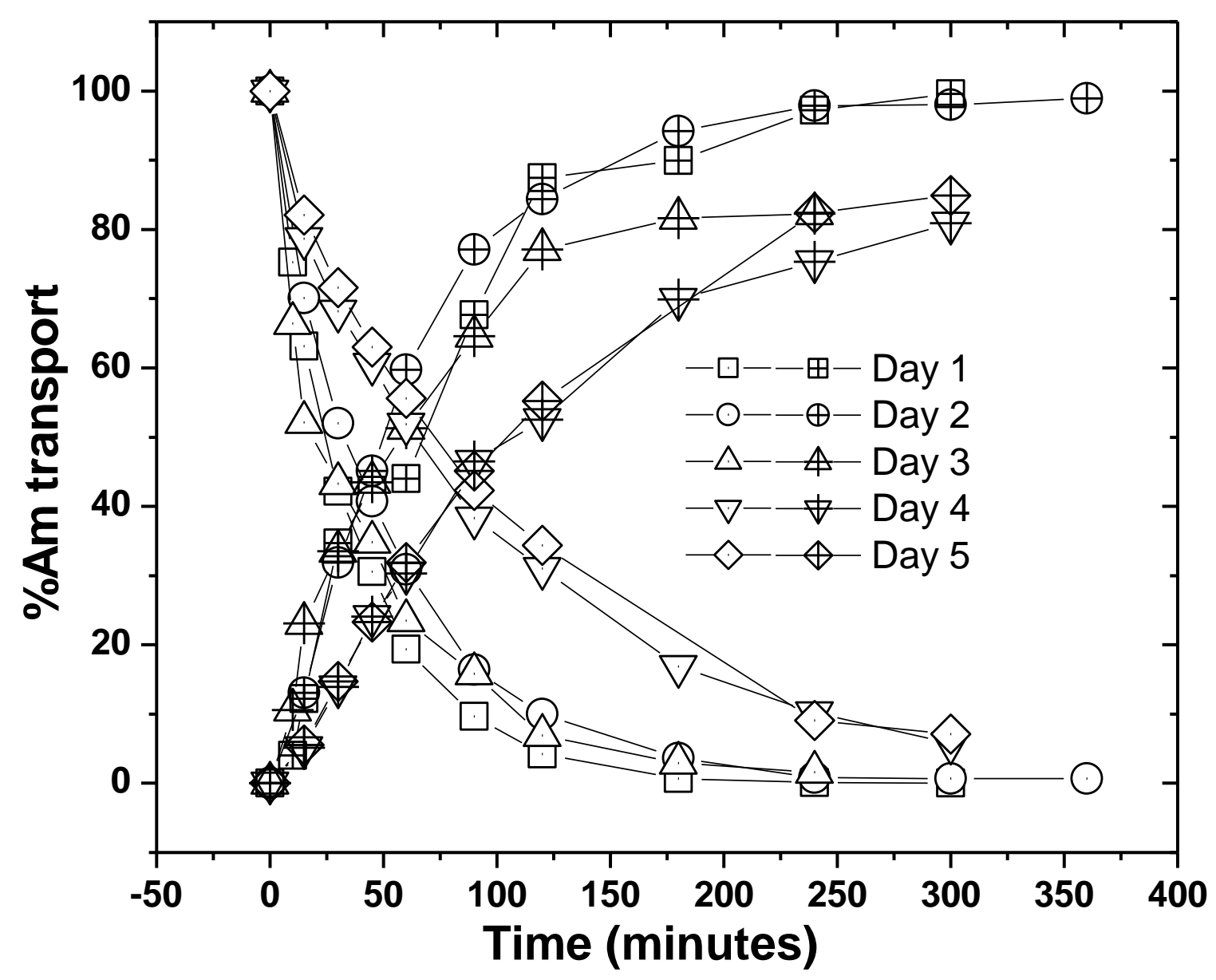


Figure S4. FT-IR spectra of pristine T-DGA containing PIM (blue) and the reused PIM (green)

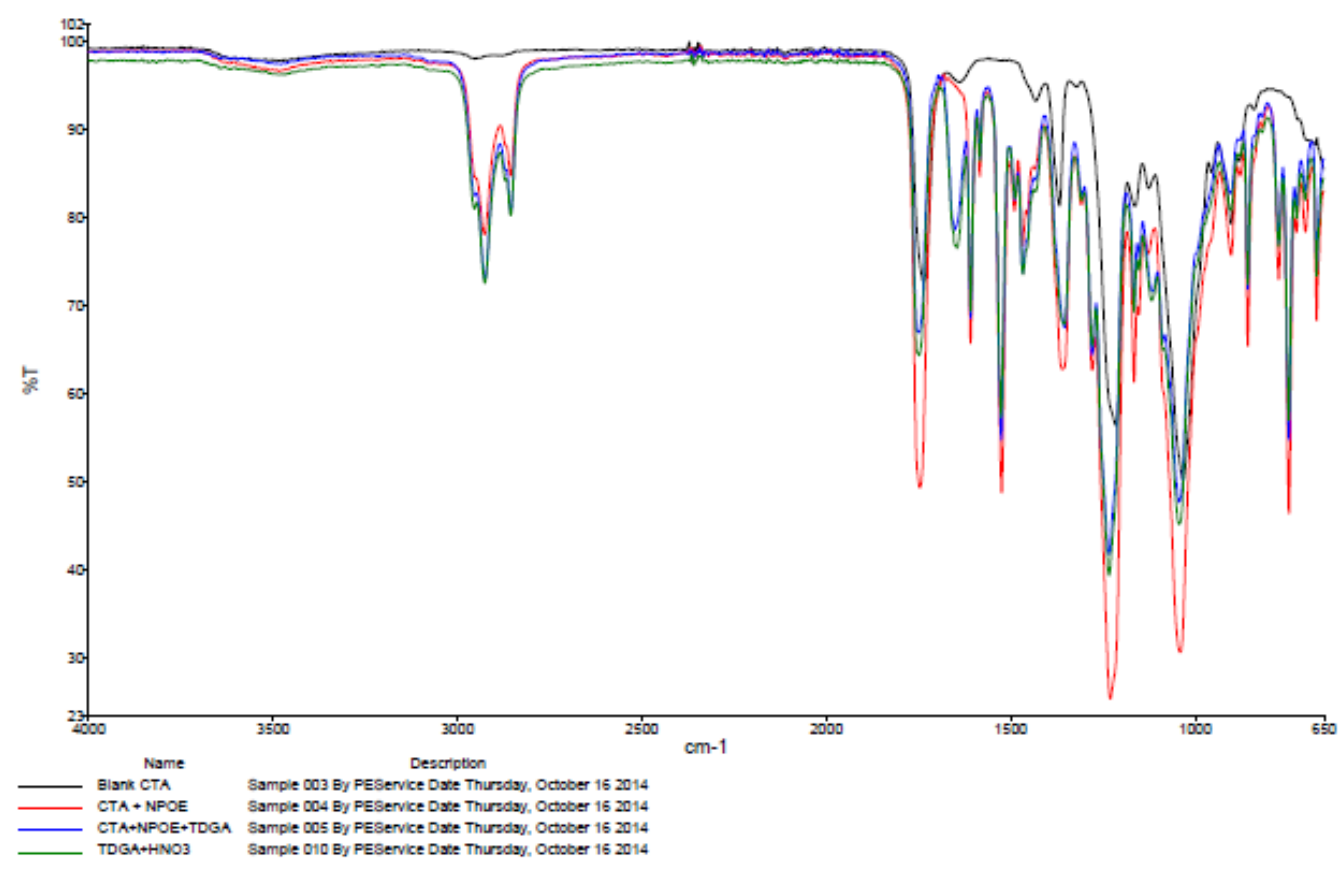

Figure S5. Chemical images of the TIMM plots obtained with a Perkin Elmer Instrument (Spotlight 300 with Spectrum 400 FTIR Spectrometer) operated in the image (transmission) mode. Pixel size: $6.25 \mu$. Scans per pixel: 16 scans; Resolution: $4 \mathrm{~cm}^{-1}$; Detector: Mercury Cadmium Telluride
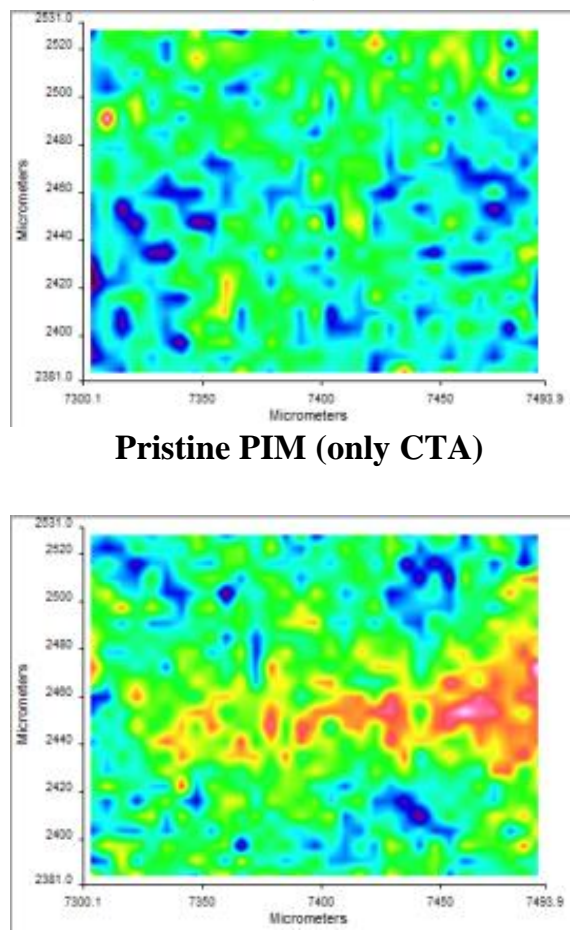

Pristine PIM (CTA+NPOE+T-DGA)
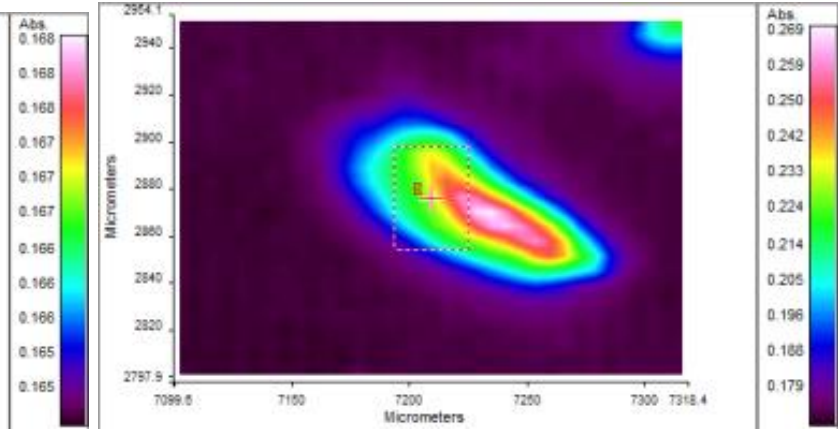

Exposed to $1 \mathrm{M} \mathrm{HNO}_{3}$ for 5 ays (only CTA)
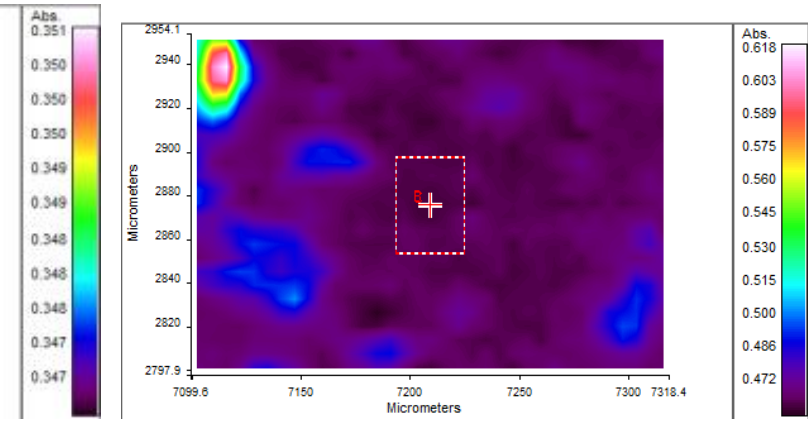

Exposed to $1 \mathrm{M} \mathrm{HNO}_{3}$ for 5 ays (CTA+NPOE+T-DGA) 


\section{AFM pictures}

Fig. S6: AFM profiles on the surface roughness of the T-DGA containing PIMs. (a) Pristine PIM; (b) PIM after 5 days contact with $1 \mathrm{M} \mathrm{HNO}_{3}$
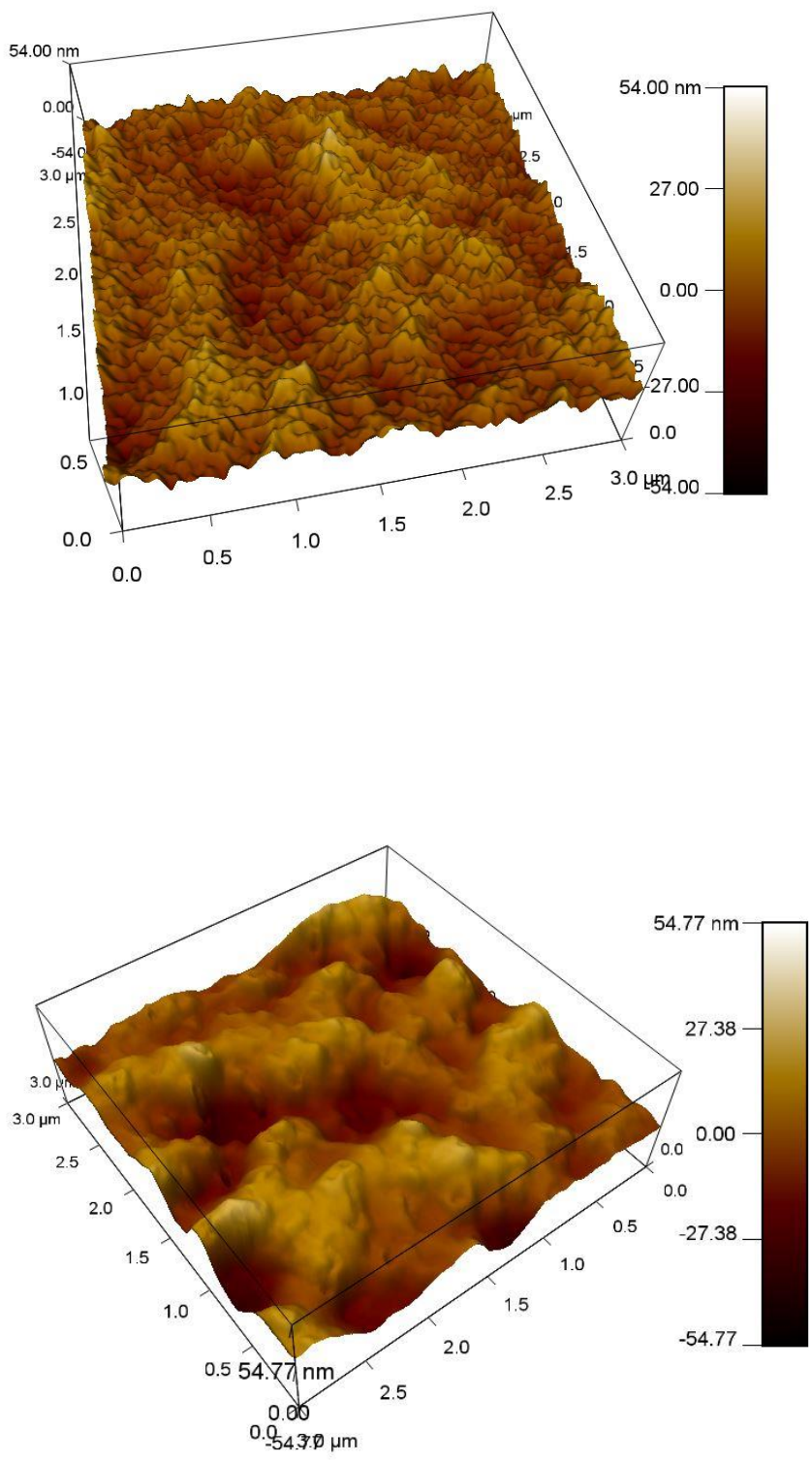